\title{
Kinetics and mechanism of the oxidation of formic and oxalic acids by quinolinium fluorochromate
}

\author{
MADHU KHURANA, PRADEEP K SHARMA and \\ KALYAN K BANERJI* \\ Department of Chemistry, JNV University, Jodhpur 342 005, India
}

MS received 19 July 1998; revised 20 January 2000

\begin{abstract}
Kinetics and mechanism of oxidation of formic and oxalic acids by quinolinium fluorochromate (QFC) have been studied in dimethylsulphoxide. The main product of oxidation is carbon dioxide. The reaction is first-order with respect to QFC. Michaelis-Menten type of kinetics were observed with respect to the reductants. The reaction is acid-catalysed and the acid dependence has the form: $k_{\mathrm{obs}}=a+b\left[\mathrm{H}^{+}\right]$. The oxidation of $\alpha$-deuterioformic acid exhibits a substantial primary kinetic isotope effect $\left(k_{\mathrm{H}} / k_{\mathrm{D}}=6.01\right.$ at $\left.303 \mathrm{~K}\right)$. The reaction has been studied in nineteen different organic solvents and the solvent effect has been analysed using Taft's and Swain's multiparametric equations. The temperature dependence of the kinetic isotope effect indicates the presence of a symmetrical cyclic transition state in the rate-determining step. Suitable mechanisms have been proposed.
\end{abstract}

Keywords. Oxidation of acids; organic acids; quinolinium fluorochromate; halochromate (VI).

\section{Introduction}

Quinolinium fluorochromate (QFC) has been used as a mild and selective oxidizing reagent in synthetic organic chemistry ${ }^{1}$. Though few reports on the mechanistic aspects of oxidation reactions of QFC are available in the literature ${ }^{2}$, there seem to be no reports on the kinetics of oxidation of oxalic acid (OA) and formic acid (FA) by QFC. We have been interested in the kinetics of the reactions of complexed $\mathrm{Cr}(\mathrm{VI})$ species and have reported the kinetics and mechanism of the oxidation of oxalic and formic acids by pyridinium fluorochromate (PFC), pyridinium chlorochromate (PCC) and pyridinium bromochromate $(\mathrm{PBC})^{3-5}$. It was observed that the oxidations by PFC and PCC presented different kinetic pictures. In this paper, we study the kinetics of oxidation of oxalic and formic acids by QFC in dimethylsulphoxide (DMSO). Mechanistic aspects are discussed.

\section{Experimental}

\subsection{Materials}

QFC and $\alpha$-deuterioformic acid $\left(\mathrm{DCO}_{2} \mathrm{H}\right.$ or DFA) were prepared by the reported methods ${ }^{1,6}$. Due to the non-aqueous nature of the medium, toluene- $p$-sulphonic acid

\footnotetext{
*For correspondence
} 
(TsOH) was used as a source of hydrogen ions. TsOH is a strong acid and in a polar solvent like DMSO it is likely to be completely ionised. Solvents were purified by the usual methods ${ }^{7}$.

\subsection{Stoichiometry}

To determine the stoichiometry, an excess of QFC $(\times 5$ or greater $)$ was reacted with the organic acid in DMSO $(100 \mathrm{ml})$ and the amount of residual QFC after the completion of reaction was measured spectrophotometrically at $365 \mathrm{~nm}$. The results indicated 1:1 stoichiometry. No quantitative determination of carbon dioxide formed was carried out.

The oxidation state of chromium in completely reduced reaction mixtures, determined by an iodometric method, was $3.98 \pm 0.35$.

\subsection{Kinetic measurements}

The reactions were followed under pseudo-first-order conditions by maintaining a large excess $(\times 15$ or greater) of the organic acid over QFC. The temperature was kept constant to $\pm 0 \cdot 1 \mathrm{~K}$. The solvent was DMSO, unless specified otherwise. The reactions were followed by monitoring the decrease in the concentration of QFC spectrophotometrically at $365 \mathrm{~nm}$ for up to $80 \%$ of the reaction. No other reactant or product has any significant

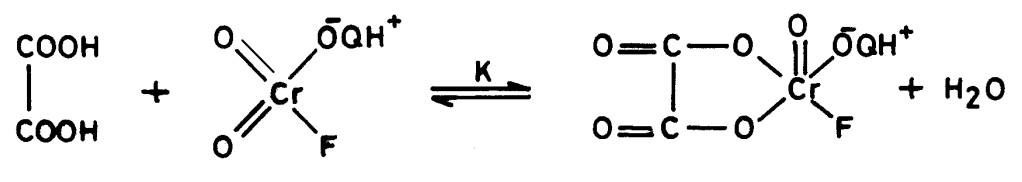

(A)
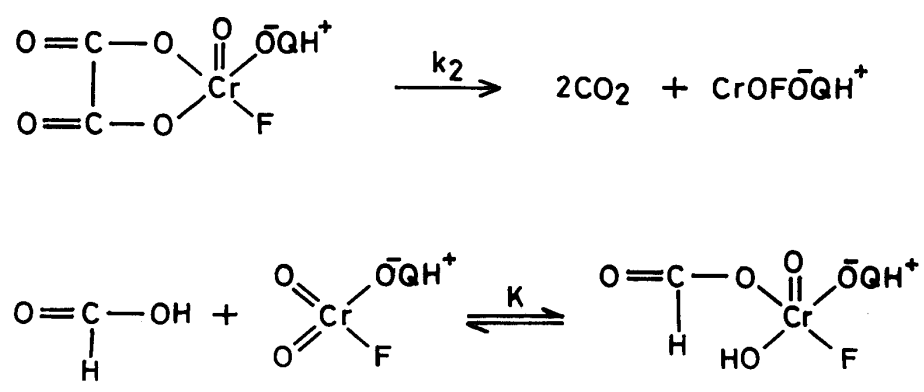

(B)

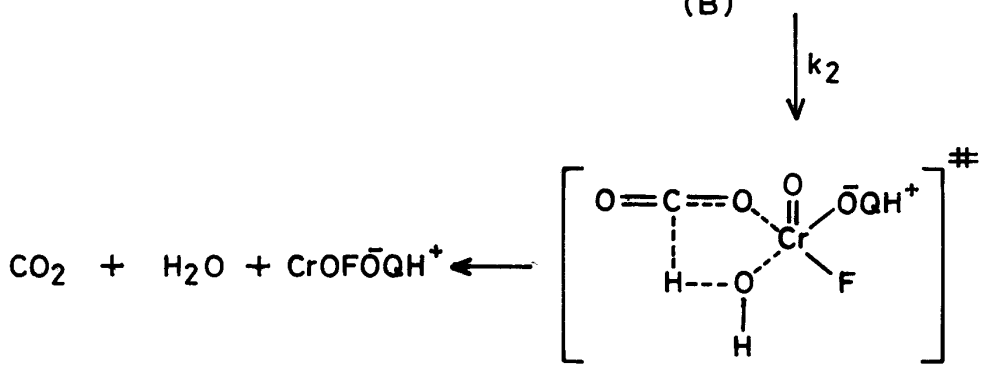

Scheme 1. 


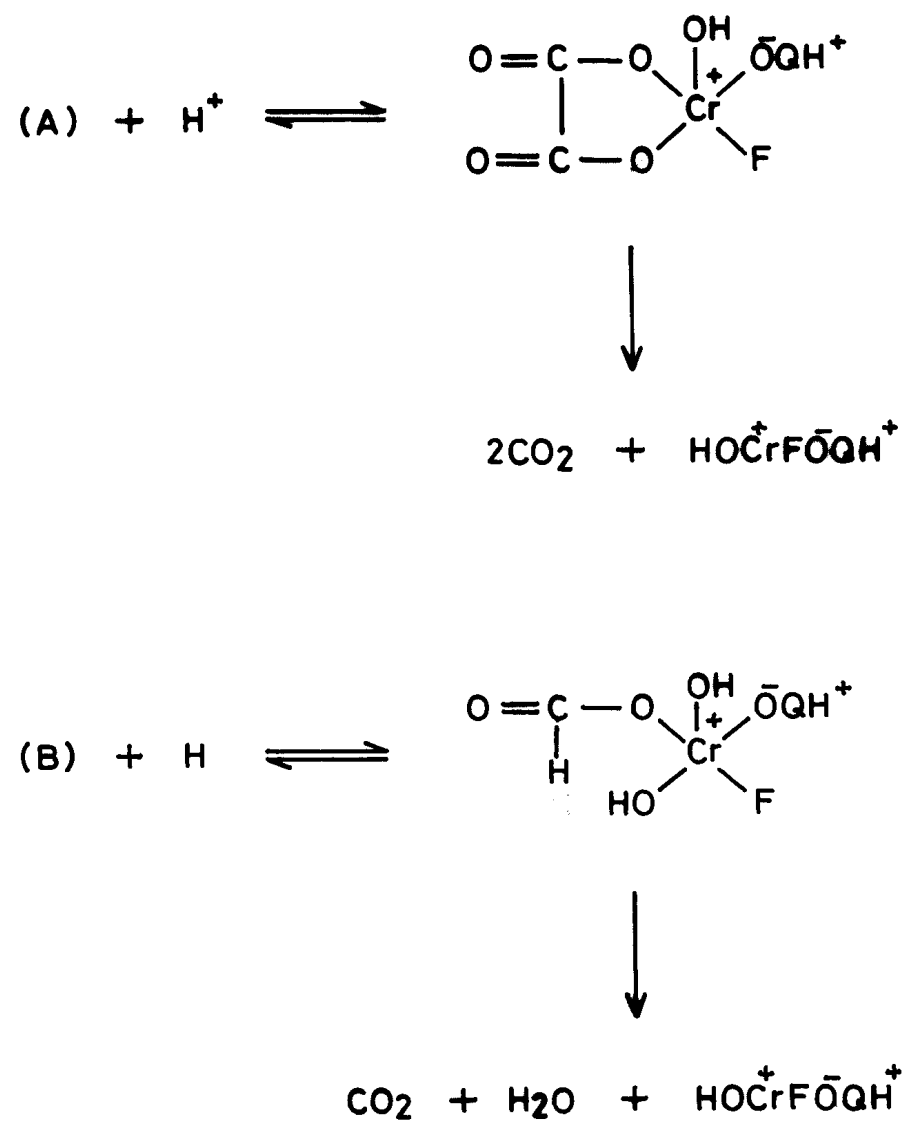

Scheme 2.

absorption at this wavelength. The pseudo-first-order rate constants, $k_{\mathrm{obs}}$, were evaluated from the linear $(r=0.995-0.999)$ plots of $\log [\mathrm{QFC}]$ against time. Duplicate kinetic runs showed that the rate constants were reproducible to within $\pm 4 \%$.

\section{Results}

\subsection{Stoichiometry}

The oxidation of organic acids leads to the formation of carbon dioxide. The stoichiometric determination indicated the following overall reactions,

$$
\begin{aligned}
& (\mathrm{COOH})_{2}+\mathrm{O}_{2} \mathrm{CrFO}^{-} \mathrm{QH}^{+} \rightarrow 2 \mathrm{CO}_{2}+\mathrm{H}_{2} \mathrm{O}+\mathrm{OCrFO}^{-} \mathrm{QH}^{+}, \\
& \mathrm{HCOOH}+\mathrm{O}_{2} \mathrm{CrFO}^{-} \mathrm{QH}^{+} \rightarrow \mathrm{CO}_{2}+\mathrm{H}_{2} \mathrm{O}+\mathrm{OCrFO}^{-} \mathrm{QH}^{+} .
\end{aligned}
$$

QFC undergoes a two-electron change. This is in accord with our earlier observations with both $\mathrm{PFC}^{3}$ and $\mathrm{QFC}^{2}$. 


\subsection{Rate laws}

The reactions were found to be first-order with respect to QFC. The reactions exhibited Michaelis-Menten type kinetics with respect to the organic acids (table 1). A plot of $1 /\left[\right.$ organic acid] vs $1 / k_{\text {obs }}$ is linear with an intercept on the rate ordinate (figure 1 ). This indicates the following overall mechanism [(3) and (4)] and the rate law (5),

$$
\begin{aligned}
& \text { organic acid }+\mathrm{QFC} \stackrel{K}{\rightleftharpoons}[\text { complex }], \\
& {\left[\text { complex } \stackrel{k_{2}}{\rightarrow}\right. \text { products, }} \\
& \left.-\mathrm{d}[\mathrm{QFC}] / \mathrm{d} t=k_{2} K[\mathrm{QFC}] \text { [organic acid }\right] /(1+K[\text { organic acid }]) .
\end{aligned}
$$

The dependence of the reaction rate on reductant concentration was studied at different temperatures and the values of $K$ and $k_{2}$ were evaluated from the double reciprocal plots. The thermodynamic and activation parameters, at $298 \mathrm{~K}$, were also calculated from the values of $K$ and $k_{2}$ respectively, at different temperatures (tables 2 and $3)$.

\subsection{Induced polymerization of acrylonitrile}

The oxidation of organic acids by QFC, in an atmosphere of nitrogen, fails to induce the polymerization of acrylonitrile. Further, the addition of acrylonitrile has no effect on the rate (table 1).

Table 1. Rate constants for the oxidation of organic acids by QFC in DMSO at $303 \mathrm{~K}$.

\begin{tabular}{lccc}
\hline & & \multicolumn{2}{c}{$10^{5} k_{\mathrm{obs}}\left(\mathrm{s}^{-1}\right)$} \\
\cline { 3 - 4 }$\left(0^{3}(\mathrm{QFC})\right.$ & $\begin{array}{c}(\text { Acid }) \\
\left(\mathrm{mol} \mathrm{dm}^{-3}\right)\end{array}$ & Oxalic acid & Formic acid \\
\hline $1 \cdot 0$ & $0 \cdot 01$ & 103 & $2 \cdot 02$ \\
$1 \cdot 0$ & $0 \cdot 02$ & 138 & $3 \cdot 88$ \\
$1 \cdot 0$ & $0 \cdot 04$ & 168 & $7 \cdot 20$ \\
$1 \cdot 0$ & $0 \cdot 12$ & 196 & $16 \cdot 8$ \\
$1 \cdot 0$ & $0 \cdot 36$ & 207 & $30 \cdot 3$ \\
$1 \cdot 0$ & $0 \cdot 48$ & 209 & $33 \cdot 6$ \\
$1 \cdot 0$ & $0 \cdot 60$ & 210 & $36 \cdot 1$ \\
$1 \cdot 0$ & $0 \cdot 12$ & $198^{\mathrm{a}}$ & $16 \cdot 3^{\mathrm{a}}$ \\
$2 \cdot 0$ & $0 \cdot 02$ & 141 & $4 \cdot 01$ \\
$4 \cdot 0$ & $0 \cdot 02$ & 136 & $3 \cdot 73$ \\
$6 \cdot 0$ & $0 \cdot 02$ & 139 & $4 \cdot 11$ \\
$8 \cdot 0$ & $0 \cdot 02$ & 140 & $3 \cdot 94$ \\
$10 \cdot 0$ & $0 \cdot 02$ & 137 & $3 \cdot 80$ \\
\hline
\end{tabular}

${ }^{\mathrm{a}}$ Contained $0.001 \mathrm{~mol} \mathrm{dm}^{-3}$ acrylonitrile 


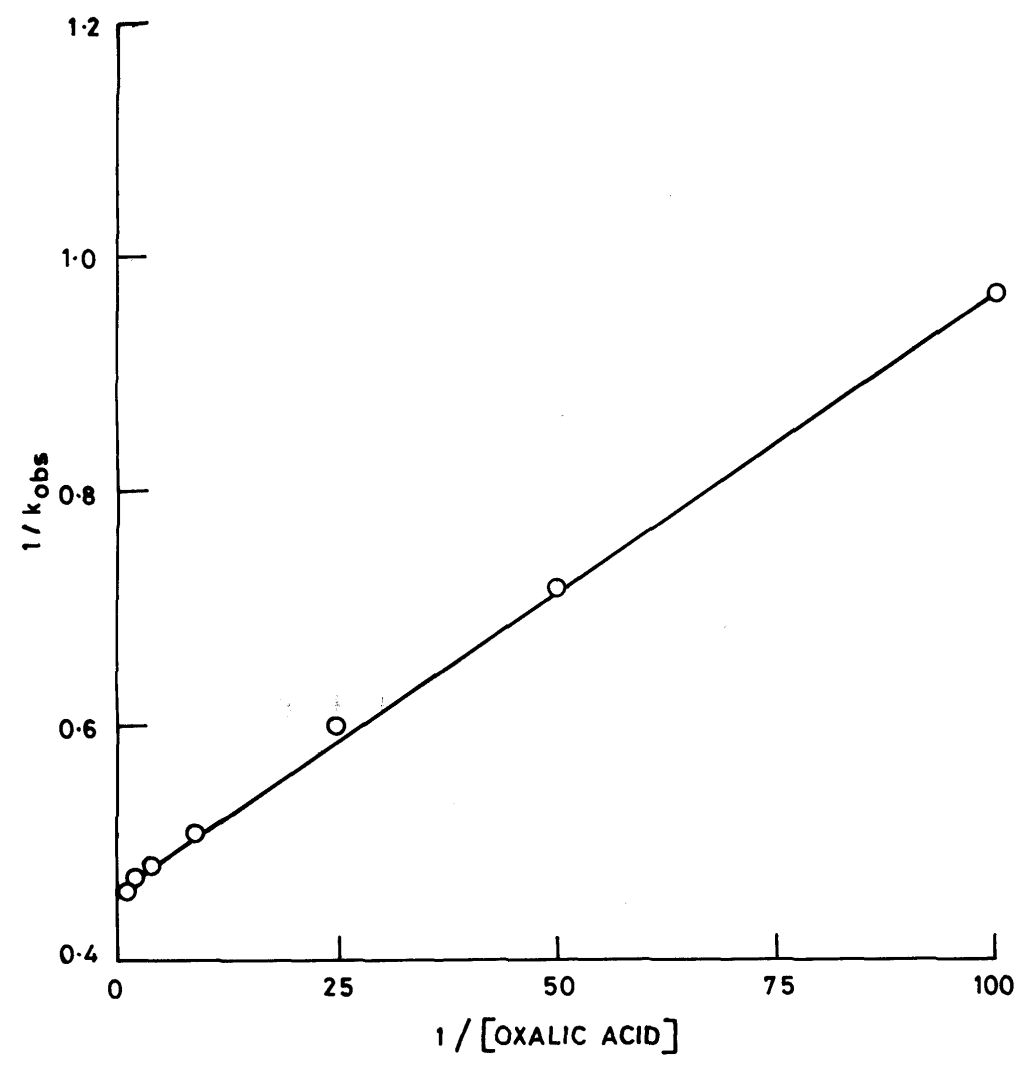

Figure 1. Plot of $1 /$ (oxalic acid) vs $1 / k_{\text {obs }}$. Reaction conditions are described in table 1.

Table 2. Formation constant and thermodynamic parameters for the organic acidQFC complexes in DMSO.

\begin{tabular}{|c|c|c|c|c|c|c|c|}
\hline \multirow[b]{2}{*}{ Acid* } & \multicolumn{4}{|c|}{$K\left(\mathrm{dm}^{3} \mathrm{~mol}^{-1}\right)$ at } & \multirow{2}{*}{$\begin{array}{c}\Delta H \\
\left(\mathrm{~kJ} \mathrm{~mol}{ }^{-1}\right)\end{array}$} & \multirow{2}{*}{$\begin{array}{c}\Delta S \\
\left(\mathrm{~J} \mathrm{~mol}^{-1} \mathrm{~K}^{-1}\right)\end{array}$} & \multirow{2}{*}{$\begin{array}{c}\Delta G \\
\left(\mathrm{~kJ} \mathrm{~mol}^{-1}\right)\end{array}$} \\
\hline & $303 \mathrm{~K}$ & $308 \mathrm{~K}$ & $313 \mathrm{~K}$ & $318 \mathrm{~K}$ & & & \\
\hline $\mathrm{OA}$ & $92 \cdot 2$ & $25 \cdot 7$ & $6 \cdot 95$ & 1.92 & $-209 \pm 2$ & $-645 \pm 7$ & $-17 \cdot 2 \pm 2 \cdot 0$ \\
\hline FA & $4 \cdot 16$ & $3 \cdot 35$ & $2 \cdot 68$ & $2 \cdot 10$ & $-38.9 \pm 0.7$ & $-107 \pm 2$ & $-6 \cdot 7 \pm 0.6$ \\
\hline DFA & $4 \cdot 28$ & $3 \cdot 45$ & $2 \cdot 78$ & $2 \cdot 25$ & $-36 \cdot 8 \pm 0 \cdot 3$ & $-102 \pm 1$ & $-6 \cdot 7 \pm 0 \cdot 2$ \\
\hline
\end{tabular}

*Abbreviations: OA - oxalic acid; FA - formic acid; DFA - deuterioformic acid

\subsection{Effect of acidity}

The reaction is catalysed by hydrogen ions. The hydrogen-ion dependence has the following form (table 4),

$$
k_{\mathrm{obs}}=a+b\left[\mathrm{H}^{+}\right]
$$


Table 3. Rate constants and activation parameters of the oxidation of organic acids by QFC in DMSO.

\begin{tabular}{|c|c|c|c|c|c|c|c|}
\hline \multirow[b]{2}{*}{ Acid } & \multicolumn{4}{|c|}{$10^{4} k_{2}\left(\mathrm{dm}^{3} \mathrm{~mol}^{-1} \mathrm{~s}^{-1}\right)$ at } & \multirow{2}{*}{$\begin{array}{c}\Delta H^{*} \\
\left(\mathrm{~kJ} \mathrm{~mol}^{-1}\right)\end{array}$} & \multirow{2}{*}{$\begin{array}{c}\Delta S^{*} \\
\left(\mathrm{~J} \mathrm{~mol}^{-1} \mathrm{~K}^{-1}\right)\end{array}$} & \multirow{2}{*}{$\begin{array}{c}\Delta G^{*} \\
\left(\mathrm{~kJ} \mathrm{~mol}^{-1}\right)\end{array}$} \\
\hline & $303 \mathrm{~K}$ & $308 \mathrm{~K}$ & $313 \mathrm{~K}$ & $318 \mathrm{~K}$ & & & \\
\hline $\mathrm{OA}$ & $21 \cdot 4$ & $31 \cdot 0$ & $43 \cdot 6$ & $62 \cdot 2$ & $54 \cdot 2 \pm 0.5$ & $-118 \pm 1$ & $89 \cdot 2 \pm 0.4$ \\
\hline FA & $5 \cdot 05$ & $7 \cdot 11$ & $10 \cdot 2$ & $13 \cdot 6$ & $50 \cdot 9 \pm 0.9$ & $-141 \pm 3$ & $92.7 \pm 0.9$ \\
\hline DFA & $0 \cdot 84$ & $1 \cdot 22$ & $1 \cdot 80$ & $2 \cdot 47$ & $55 \cdot 6 \pm 0 \cdot 8$ & $-140 \pm 3$ & $97 \cdot 2 \pm 0.7$ \\
\hline$k_{\mathrm{H}} / k_{\mathrm{D}}$ & $6 \cdot 01$ & $5 \cdot 82$ & $5 \cdot 66$ & $5 \cdot 50$ & & & \\
\hline
\end{tabular}

Abbreviations as in table 2

Table 4. Dependence of the reaction rate on hydrogen-ion concentration. $[\mathrm{OA}]$ and $[\mathrm{FA}]=0.02 \mathrm{~mol} \mathrm{dm}^{-3},[\mathrm{QFC}] 0.001 \mathrm{~mol} \mathrm{dm}^{-3}$; temperature $303 \mathrm{~K}$; solvent: DMSO

\begin{tabular}{lcccccc}
\hline & \multicolumn{6}{c}{ at $(\mathrm{TsOH}) /\left(\mathrm{mol} \mathrm{dm}^{-3}\right)$} \\
\cline { 2 - 7 } $\begin{array}{l}\text { Acid } \\
10^{5} k_{\text {obs }}\left(\mathrm{s}^{-1}\right)\end{array}$ & $0 \cdot 10$ & 0.20 & 0.40 & 0.60 & 0.80 & 1.00 \\
\hline OA & 186 & 237 & 325 & 437 & 526 & 610 \\
FA & 5.23 & 6.66 & 9.15 & 12.3 & 14.8 & 17.1 \\
\hline
\end{tabular}

The values of $a$ and $b$ for oxalic acid, are $1.41 \pm 0.06 \times 10^{-3} \mathrm{~s}^{-1}$ and $4.76 \pm 0.09 \times 10^{-3}$ $\mathrm{mol}^{-1} \mathrm{dm}^{3} \mathrm{~s}^{-1}$ respectively $\left(r^{2}=0.9984\right)$. The corresponding values for the oxidation of formic acid are $3.97 \pm 0.20 \times 10^{-5} \mathrm{~s}^{-1}$ and $1.34 \pm 0.03 \times 10^{-4} \mathrm{~mol}^{-1} \mathrm{dm}^{3} \mathrm{~s}^{-1}\left(r^{2}=0.9981\right)$.

\subsection{Kinetic isotope effect}

To ascertain the importance of the cleavage of the $\alpha-\mathrm{C}-\mathrm{H}$ bond in the rate-determining step, the oxidation of DFA was studied. The results, recorded in tables 2 and 3, show that while the formation constant $K$, for the ordinary and deuteriated formic acids have almost identical values, the rate constant for the decomposition of the complex, $k_{2}$, exhibits a substantial primary kinetic isotope effect $\left(k_{\mathrm{H}} / k_{\mathrm{D}}=6.01\right.$ at $\left.303 \mathrm{~K}\right)$.

\subsection{Solvent effect}

The oxidation of formic acid was studied in 19 different organic solvents. The choice of solvents was limited due to the solubility of QFC and its reaction with primary and secondary alcohols. There was no reaction with the solvents chosen. The kinetics were similar in all the solvents. The values of $K$ and $k_{2}$ are recorded in table 5.

\section{Discussion}

\subsection{Solvent effect}

The formation constant of the intermediate complex, $K$, did not vary much with the solvent but the rate constant, $k_{2}$, showed much variation in values with different solvents. 
Table 5. Formation constants and rate constants for the decomposition of formic acid-QFC complex in different solvents at $303 \mathrm{~K}$.

\begin{tabular}{lcclcc}
\hline Solvents & $\begin{array}{c}K \\
\left.(\mathrm{dm} \mathrm{mol})^{-1}\right)\end{array}$ & $\begin{array}{c}10^{5} k_{2} \\
\left(\mathrm{~s}^{-1}\right)\end{array}$ & \multicolumn{1}{c}{ Solvents } & $\begin{array}{c}K \\
\left(\mathrm{dm} \mathrm{mol}^{-1}\right)\end{array}$ & $\begin{array}{c}10^{5} k_{2} \\
\left(\mathrm{~s}^{-1}\right)\end{array}$ \\
\hline Chloroform & $4 \cdot 03$ & $15 \cdot 8$ & Acetic acid & $4 \cdot 66$ & $3 \cdot 98$ \\
1,2-Dichloromethane & $4 \cdot 05$ & $14 \cdot 5$ & Cyclohexane & $4 \cdot 83$ & $0 \cdot 22$ \\
Dichloromethane & $4 \cdot 27$ & $16 \cdot 2$ & Toluene & $4 \cdot 96$ & $2 \cdot 63$ \\
DMSO & 4.16 & $50 \cdot 5$ & Acetophenone & $3 \cdot 60$ & $15 \cdot 4$ \\
Acetone & 5.00 & $11 \cdot 2$ & THF & 4.33 & $4 \cdot 89$ \\
DMF & 4.58 & $24 \cdot 5$ & t-Butyl alcohol & $5 \cdot 17$ & $6 \cdot 46$ \\
Butanone & $4 \cdot 12$ & $8 \cdot 13$ & 1,4-Dioxane & $4 \cdot 82$ & $5 \cdot 50$ \\
Benzene & $5 \cdot 14$ & $3 \cdot 24$ & Carbon disulphide & $4 \cdot 14$ & $1 \cdot 17$ \\
Ethylacetate & $3 \cdot 66$ & 3.98 & 1,2-Dimethoxyethane & $4 \cdot 37$ & $2 \cdot 75$ \\
Nitrobenzene & $3 \cdot 82$ & $17 \cdot 4$ & & & \\
\hline
\end{tabular}

The rate constants, $k_{2}$, in eighteen solvents $\left(\mathrm{CS}_{2}\right.$ was not considered, as the complete range of solvent parameters was not available) were correlated in terms of the linear solvation energy relationship of Kamlet $e a^{8}{ }^{8}$,

$$
\log k_{2}=A_{0}+p \pi^{*}+b \beta+a \alpha \text {. }
$$

In this equation, $\pi^{*}$ represents the solvent polarity, $\beta$ the hydrogen bond acceptor basicities and $\alpha$ the hydrogen bond donor acidity. $A_{0}$ is the intercept term. It may be mentioned here that out of the 18 solvents, 12 have a value of zero for $\alpha$. The results of correlation analyses in terms of (7), a biparametric equation involving $\pi^{*}$ and $\beta$, and separately with $\pi^{*}$ and $\beta$ are given below.

$$
\begin{aligned}
& \log k_{2}=-5 \cdot 56+2 \cdot 03( \pm 0 \cdot 20) \pi^{*}+0 \cdot 13( \pm 0 \cdot 17) \beta+0 \cdot 40( \pm 0 \cdot 16) \alpha, \\
& R^{2}=0 \cdot 8933 ; \mathrm{sd}=0 \cdot 19 ; n=18 ; \psi=0 \cdot 26, \\
& \log k_{2}=-5 \cdot 47+1 \cdot 88( \pm 0 \cdot 23) \pi^{*}+0 \cdot 27( \pm 0 \cdot 18) \beta, \\
& R^{2}=0 \cdot 8440 ; \mathrm{sd}=0 \cdot 22 ; n=18 ; \psi=0 \cdot 30, \\
& \log k_{2}=-5 \cdot 41+1.96( \pm 0 \cdot 22) \pi^{*}, \\
& r^{2}=0 \cdot 8217 ; \mathrm{sd}=0.22 ; n=18 ; \psi=0 \cdot 31, \\
& \log k_{2}=-5 \cdot 63+0 \cdot 61( \pm 0 \cdot 42) \beta, \\
& r^{2}=0 \cdot 1181 ; \mathrm{sd}=0.49 ; n=18 ; \psi=0 \cdot 83 .
\end{aligned}
$$

Here $n$ is the number of data points and $\psi$ is the Exner statistical parameter ${ }^{9}$.

Kamlet's ${ }^{8}$ triparametric equation explains $\approx 89 \%$ of the effect of solvent on the oxidation. However, by Exner's criterion ${ }^{9}$ the correlation is not even satisfactory (cf. (8)). The major contribution is of solvent polarity. It alone accounts for $\approx 82 \%$ of the data. Both $\beta$ and $\alpha$ play relatively minor roles. 
Data on the solvent effect were also analysed in terms of Swain's equation ${ }^{10}$ of the cation- and anion-solvating concept of the solvents

$$
\log k_{2}=a A+b B+C \text {. }
$$

Here, $A$ represents the anion-solvating power of the solvent and $B$ the cation-solvating power. $C$ is the intercept term. $(A+B)$ is postulated to represent the solvent polarity. The rates in different solvents were analysed in terms of (12), separately with both $A$ and $B$ and with $(A+B)$.

$$
\begin{aligned}
& \log k_{2}=1 \cdot 54( \pm 0 \cdot 02) A+1 \cdot 83( \pm 0 \cdot 01) B-5 \cdot 79, \\
& R^{2}=0.9992 ; \mathrm{sd}=0 \cdot 02 ; n=19 ; \psi=0 \cdot 02, \\
& \log k_{2}=1 \cdot 28( \pm 0 \cdot 60) A-4 \cdot 54, \\
& r^{2}=0 \cdot 2107 ; \mathrm{sd}=0 \cdot 49 ; n=19 ; \psi=0 \cdot 76, \\
& \log k_{2}=1 \cdot 71( \pm 0 \cdot 27) \mathrm{B}-5 \cdot 29, \\
& r^{2}=0 \cdot 6978 ; \mathrm{sd}=0 \cdot 30 ; n=19 ; \psi=0 \cdot 42 \\
& \log k_{2}=1 \cdot 73 \pm 0 \cdot 04(A+B)-5 \cdot 78 \\
& r^{2}=0.9918 ; \mathrm{sd}=0.05 ; n=19 ; \psi=0 \cdot 07
\end{aligned}
$$

The rates of decomposition of the complex in different solvents showed excellent correlation in Swain's equation (cf. (13)) with the cation-solvating power playing the major role. In fact, cation-solvation alone accounts for $\approx 70 \%$ of the data. Correlation with the anion-solvating power was very poor. Solvent polarity, represented by $(A+B)$, also accounted for $\approx 99 \%$ of the data. In view of the fact that solvent polarity is able to account for $\approx 99 \%$ of the data, an attempt was made to correlate the rate with the relative permittivity of the solvent. However, a plot of log (rate) against the inverse of the relative permittivity is not linear $\left(r^{2}=0.5949 ; \mathrm{sd}=0.39 ; \psi=0.49\right)$.

\section{Mechanism}

The presence of a substantial kinetic isotopic effect confirmed that an $\alpha-\mathrm{C}-\mathrm{H}$ bond is cleaved in the rate-determining step. The observed kinetics indicate the formation of an intermediate complex in a rapid pre-equilibrium. However, the highly unfavourable entropy term obtained in the complex formation of oxalic acid-QFC reaction suggests that oxalic acid acts as a bidentate ligand and forms a cyclic intermediate complex. In the chromic acid oxidation also, the formation of a cyclic anhydride intermediate, oxalyl chromate, has been postulated ${ }^{11}$. The value of formation constant, $9.5 \mathrm{dm}^{3} \mathrm{~mol}^{-1}$, reported by Hassan and Rocek ${ }^{11}$ compares favourably with the values obtained in this investigation. The absence of any effect of a radical scavenger, acrylonitrile, indicates that a hydrogen abstraction mechanism, giving rise to free radicals, is unlikely. 
In formic acid oxidation, the cation-solvating power of the solvents plays a relatively more important role. Therefore, formation of an electron-deficient carbon centre in the transition state is indicated. Thus the decomposition of QFC-formic acid complex may involve hydride ion transfer via an anhydride intermediate.

Involvement of a concerted cyclic process is supported by a study of the temperature dependence of the kinetic isotope effect ${ }^{12}$. The data for protio- and deuterio-formic acids when fitted in the familiar expression $k_{\mathrm{H}} / k_{\mathrm{D}}=A_{\mathrm{H}} / A_{\mathrm{D}} \exp \left(-\Delta H^{*} / R T\right)$ show direct correspondence with the properties of a symmetrical transition state in which the differences in the activation energies for the protio and deuterio compounds are equal to the differences in the zero point energies of the corresponding $\mathrm{C}-\mathrm{H}$ and $\mathrm{C}-\mathrm{D}$ bonds $\left(\approx 4.5 \mathrm{~kJ} \mathrm{~mol}^{-1}\right)$ and the entropies of the activation of the respective reactions are almost equal ${ }^{13,14}$.

The observed dependence on the hydrogen-ion concentration in both the reactions shows there is an additional acid-catalysed pathway. This may be attributed to rapid reversible protonation of the anhydride, with the protonated anhydride decomposing at a rate higher than the decomposition of the unprotonated anhydride.

In the oxidation of these acids by $\mathrm{QFC}, \mathrm{PFC}^{3}$ and $\mathrm{PBC}^{5}$, Michaelis-Menten-type kinetics, with respect to the reductants, are obtained, but with $\mathrm{PCC}^{7}$ the reactions are firstorder. It is possible that the values of formation constants for the reductant-PCC complexes are very low. This results in the observation of second-order kinetics. No explanation of the difference is available presently. Kinetic isotope effects, solvent effects and dependence on hydrogen ion concentration are similar in all these reactions, for which essentially similar mechanisms have been proposed.

\section{Acknowledgement}

The authors thank the University Grants Commission, New Delhi for financial support.

\section{References}

1. Murugesan V and Pandurangan A 1992 Indian J. Chem. B31 377

2. Murugesan V and Pandurangan A 1995 React. Kinet. Catal. Lett. 54173

3. Asopa R, Mathur A and Banerji K K 1992 J. Chem. Res. (S)152, (M)1117

4. Varshney S, Kothari S and Banerji K K 1992 J. Chem. Res. (S)356, (M)2901

5. Rathore S, Sharma P K and Banerji K K 1994 J. Chem. Res. (S)504

6. Wiberg K B and Stewart R 1956 J. Am. Chem. Soc. 781214

7. Perrin D D, Armarego L and Perrin D R 1966 Purification of organic compounds (Oxford: Pergamon)

8. Kamlet M J, Abboud J L M, Abraham M H and Taft R W 1983 J. Org. Chem. 48 2877, and references cited therein

9. Exner O 1966 Collect. Czech. Chem. Commun. 313222

10. Swain C G, Swain M S, Powel A L and Alunni S 1983 J. Am. Chem. Soc. 105502

11. Hassan F and Rocek J 1972 J. Am. Chem. Soc. 929073

12. Kwart H and Nickel J H 1973 J. Am. Chem. Soc. 953394

13. Kwart H and Latimer M C J. Am. Chem. Soc. 933770

14. Kwart H and Slutsky J 1972 J. Chem. Soc., Chem. Commun. 1182 